\title{
Xenopus polo-like kinase Plx1 regulates XErp1, a novel inhibitor of APC/C activity
}

\author{
Andreas Schmidt, ${ }^{1}$ Peter I. Duncan, ${ }^{2,4}$ Nadine R. Rauh, ${ }^{1}$ Guido Sauer, ${ }^{2}$ Andrew M. Fry, ${ }^{3}$ \\ Erich A. Nigg, ${ }^{2}$ and Thomas U. Mayer ${ }^{1,5}$ \\ ${ }^{1}$ Chemical Biology, Independent Research Group, and ${ }^{2}$ Department of Cell Biology, Max-Planck-Institute of Biochemistry, \\ 82152 Martinsried, Germany; ${ }^{3}$ Department of Biochemistry, University of Leicester, Leicester LE1 7RH, UK
}

\begin{abstract}
Metaphase-to-anaphase transition is a fundamental step in cell cycle progression where duplicated sister-chromatids segregate to the future daughter cells. The anaphase-promoting complex/cyclosome (APC/C) is a highly regulated ubiquitin-ligase that triggers anaphase onset and mitotic exit by targeting securin and mitotic cyclins for destruction. It was previously shown that the Xenopus polo-like kinase Plx1 is essential to activate APC/C upon release from cytostatic factor (CSF) arrest in Xenopus egg extract. Although the mechanism by which Plx1 regulates APC/C activation remained unclear, the existence of a putative APC/C inhibitor was postulated whose activity would be neutralized by Plx1 upon CSF release. Here we identify XErp1, a novel Plx1-regulated inhibitor of APC/C activity, and we demonstrate that XErp1 is required to prevent anaphase onset in CSF-arrested Xenopus egg extract. Inactivation of XErp1 leads to premature APC/C activation. Conversely, addition of excess XErp1 to Xenopus egg extract prevents APC/C activation. Plx1 phosphorylates XErp1 in vitro at a site that targets XErp1 for degradation upon CSF release. Thus, our data lead to a model of APC/C activation in Xenopus egg extract in which Plx1 targets the APC/C inhibitor XErp1 for degradation.
\end{abstract}

[Keywords: Cell cycle; anaphase-promoting complex/cyclosome; Xenopus; polo-like kinase; cytostatic factor; mitotic exit]

Received August 9, 2004; revised version accepted December 16, 2004.

Before fertilization, vertebrate eggs are arrested in metaphase of meiosis II by a biochemical activity that was named cytostatic factor (CSF) in a seminal publication over 30 years ago (Masui and Markert 1971). CSF was defined as an activity present in the cytoplasm of frog eggs arrested in metaphase of meiosis II that when injected into one cell of a two-cell embryo produced a cleavage arrest in the injected cell. Since then, numerous attempts have been made to identify the molecular nature of CSF in vertebrate eggs and to dissect the mechanism underlying the meiotic metaphase arrest. Fertilization of the egg causes a transient rise in free intracellular calcium mediating CSF release and thus anaphase-promoting complex/cyclosome (APC/C) activation. Active $\mathrm{APC} / \mathrm{C}$ mediates the degradation of securin and cyclin B and thereby promotes anaphase onset (Lorca et al. 1993; Murray 2004). Extract from CSF-arrested Xenopus eggs faithfully recapitulates many morphological and biochemical events associated with release from CSF arrest upon addition of calcium ions.

${ }^{4}$ Present address: Nestlé Research Center, Vers-chez-les-Blanc, PO Box 44, CH-1000 Lausanne 26, Switzerland.

${ }^{5}$ Corresponding author.

E-MAIL mayer@biochem.mpg.de; FAX 49-89-8578-3138.

Article and publication are at http://www.genesdev.org/cgi/doi/10.1101/ gad.320705.
Data from various research groups have firmly established a role for the germ-cell specific c-Mos kinase in establishing CSF activity (Sagata et al. 1989; Tunquist and Maller 2003). c-Mos mediates its effect by activating a mitogen-activated protein kinase (MAPK) cascade (Kosako et al. 1994) leading to the phosphorylation and thereby activation of the protein kinase $\mathrm{p} 90^{\text {Rsk }}$ (Sturgill et al. 1988). Components of the spindle assembly checkpoint seem to be the downstream targets of the c-Mos/ $\mathrm{MAPK} / \mathrm{p} 90^{\mathrm{Rsk}}$ pathway, because the checkpoint proteins Mad1, Mad2, and Bub1 are required to mediate the c-Mos-initiated CSF arrest in Xenopus egg extract (Schwab et al. 2001; Tunquist et al. 2002, 2003). However, the c-Mos/MAPK/p90 Rsk pathway is not the only activity contributing to CSF arrest. For instance, it has been shown that cyclin-dependent kinase 2/cyclin $\mathrm{E}$ (Cdk2/cyclin E) could mediate CSF arrest in the absence of c-Mos kinase (Tunquist et al. 2002), suggesting that $\mathrm{Cdk} 2 /$ cyclin $\mathrm{E}$ and c-Mos/MAPK/p90 ${ }^{\text {Rsk }}$ represent independent pathways both needed for effective APC/C inhibition. Furthermore, recent work identified a putative vertebrate homolog of the Drosophila regulator of cyclin A1 (Dong et al. 1997), Emil (early mitotic inhibitor 1), as an additional independent element contributing to CSF activity in Xenopus eggs (Reimann et al. 2001a; Reimann and Jackson 2002). Emil, a conserved F-box protein with a putative zinc-binding region (ZBR) has been shown to 
bind to the APC/C activator CDC20 (Reimann et al. 2001a) thereby preventing APC/C activation.

Polo-like kinases (Plks) play a pivotal role in regulating progression through $\mathrm{M}$ phase in all eukaryotic cells (Barr et al. 2004). Evidence from different model organisms suggests that Plk1 activity is required for the timely activation of the APC/C at anaphase onset (Descombes and Nigg 1998; Shirayama et al. 1998; Donaldson et al. 2001). These data are supported by studies showing that Plk1, like Cdk1, can phosphorylate specific APC/C subunits in vitro (Kotani et al. 1998; Golan et al. 2002). However, it is still an open question whether the phosphorylation of APC/C by Plk1 is a prerequisite for APC/C's ubiquitin ligase activity. For example, whereas Golan et al. (2002) reported that both Cdk1 and Plk1 can stimulate APC/C ubiquitin ligase activity, recent data suggest that the phosphorylation of APC/C by Cdk1 but not by Plk1 increases the activity of APC/C in vitro (Kraft et al. 2003). Although the biological relevance of APC/C phosphorylation by Plk1 remains to be determined, experiments in Xenopus egg extract have clearly established a role of $\mathrm{Plx} 1$ in activating the APC/C in response to calcium (Descombes and Nigg 1998) and in maintaining the APC/C in its active form (Brassac et al. 2000). Xenopus egg extract depleted of endogenous Plx1 or supplemented with catalytically inactive $\mathrm{Plx} 1^{\mathrm{N} 172 \mathrm{~A}}$ could not activate the APC/C upon calcium addition and therefore did not enter interphase (Descombes and Nigg 1998). Although that study did not address the mechanism by which Plx1 allows the destruction of APC/C targets upon CSF release, the existence of a putative Plx1-regulated APC/C inhibitor was proposed (Descombes and Nigg 1998). Such an inhibitor would be expected to be active in CSF extract but would be inactivated by Plx1 upon fertilization.

We have identified XErp1 as a novel Plx1-interacting protein. XErp1 is a conserved F-box protein with a putative ZBR at its $\mathrm{C}$ terminus. XErp1 is required to maintain CSF-mediated metaphase arrest in Xenopus egg extract. Excess XErp1 can prevent calcium-induced CSF release in Xenopus egg extract. In vitro, XErpl can directly inhibit the ubiquitin ligase activity of APC/C $\mathrm{CDC}^{\mathrm{CD} O}$. Plx1 phosphorylates XErp1 at a site in vitro that targets XErp1 for degradation at anaphase onset in Xenopus egg extract. The activity of XErp1 is required to mediate the dominant-negative effect of the Plx1 polo-box domain (PBD) on CSF release. Our data lead to a model in which Plx1mediated degradation of XErpl is a prerequisite for APC/C activation upon CSF release in Xenopus egg extract.

\section{Results}

Identification of XErp1, a novel interactor of Plx1

To identify the hypothetical Plx1-regulated APC/C inhibitor proposed by Descombes and Nigg (1998) we performed a yeast-two hybrid screen. To this end we fused full-length catalytically inactive $\mathrm{Plx} 1\left(\mathrm{Plx}^{\mathrm{N} 172 \mathrm{~A}}\right)$ to the DNA-binding domain of Gal4 and used this bait to screen a Xenopus oocyte cDNA library. DNA sequence analysis of the 25 interactors identified revealed that two independent clones were derived from the same gene. The full-length cDNA of these two clones encoded an F-box protein of 651 amino acids which we named XErp1, for Xenopus Emi1-related protein 1 (formerly referred to as Pxp17, acc. no. AAN76807). Protein sequence analyses revealed that XErp1 contains a putative ZBR at its $\mathrm{C}$ terminus (Fig. 1A,B).

The cDNA sequence of XErp1 is $97 \%$ identical to the cDNA sequence of Fbx26, which was previously identified as a novel Xenopus F-box containing protein (ReganReimann et al. 1999). However, unlike XErp1 the protein predicted by the $F b \times 26$ cDNA sequence does not contain a putative ZBR. To resolve the discrepancy between the two predicted protein sequences, we performed microsequence analyses of endogenous XErp1 protein immunopurified from Xenopus egg extract. Liquid chromatography-tandem mass spectrometry (LC-MS/MS) analyses identified several peptide sequences that match the protein sequence predicted by the XErp1 ORF but not by the Fbx26 ORF (Fig. 1B). Most of the discrepancies between the identified peptide sequences and the $F b \times 26$ ORF arise from frame shifts within the $F b \times 26$ cDNA sequence, suggesting that the reported $F b \times 26$ sequence is incorrect. In light of these data we conclude that the XErp1 and Fbx26 cDNAs encode identical proteins and that the XErp1 ORF reported here provides the correct sequence. The $\mathrm{N}$ terminus of XErp1 displays no significant sequence homology to known proteins, whereas the $\mathrm{C}$ terminus of XErp1 shows significant sequence similarity to Emil (Fig. 1A), a known APC/C inhibitor.

Affinity-purified antibodies raised against an $\mathrm{N}$-terminal moiety of XErp1 fused to a six-histidine tag $\left(\mathrm{His}_{6}\right.$ XErp $1^{105-374}$ ) reacted with a polypeptide of an apparent molecular mass of $\sim 90 \mathrm{kDa}$ in immunoblots of extract from CSF-arrested Xenopus eggs (Fig. 1C, left panel). To confirm that the detected polypeptide is XErp1, we used the affinity-purified antibody to probe immunoblots of in vitro translated (IVT), ${ }^{35}$ S-labeled XErp1, Emi1, and unprogrammed wheat-germ extract (WGE). As shown in the left panel of Figure 1C, the antibodies recognized IVT XErp1 but failed to detect IVT Emil or any protein to a substantial degree in the unprogrammed WGE. The reason for the observed difference in the electrophoretic mobility between the endogenous XErp1 present in CSF extract and IVT XErp1 will be addressed below. Autoradiographic analysis of the immunoblots confirmed that both ${ }^{35}$ S-labeled IVT products, XErp1 and Emi1, were expressed at similar levels in the WGE (Fig. 1C, middle panel). Antibodies preincubated with the antigen $\mathrm{His}_{6}$ XErp $1^{105-374}$ completely failed to detect XErp1 in CSF extract and IVT XErp1 (Fig. 1C, right panel). Taken together, these data demonstrate that our antibody specifically recognizes XErp1.

\section{XErp1 is degraded upon CSF release}

If XErp1 represents the postulated APC/C inhibitor, then XErp1 should be active in CSF extract and should be 
Schmidt et al.

inactivated upon CSF release. Immunoblot analysis showed that XErp1 is present in CSF extract but rapidly degraded upon calcium addition (Fig. 2A). Microscopic examination of the extract revealed that the chromatin decondensed upon calcium addition, confirming that the extract had entered interphase (Fig. 2A). When CSF ex-

A

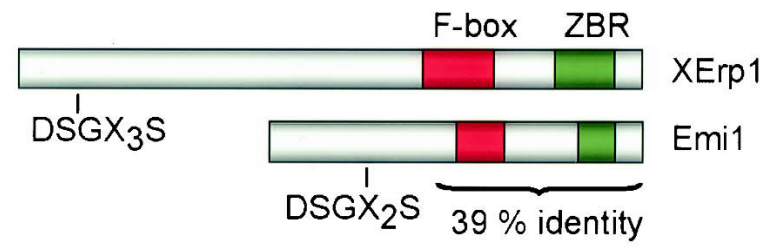

B

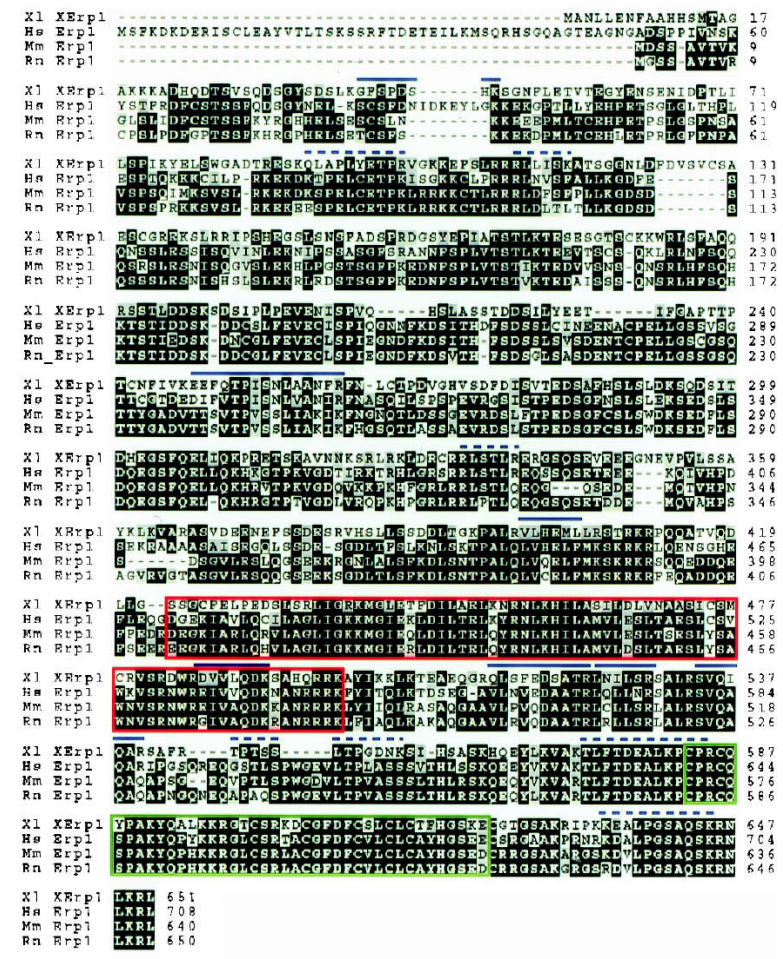

C

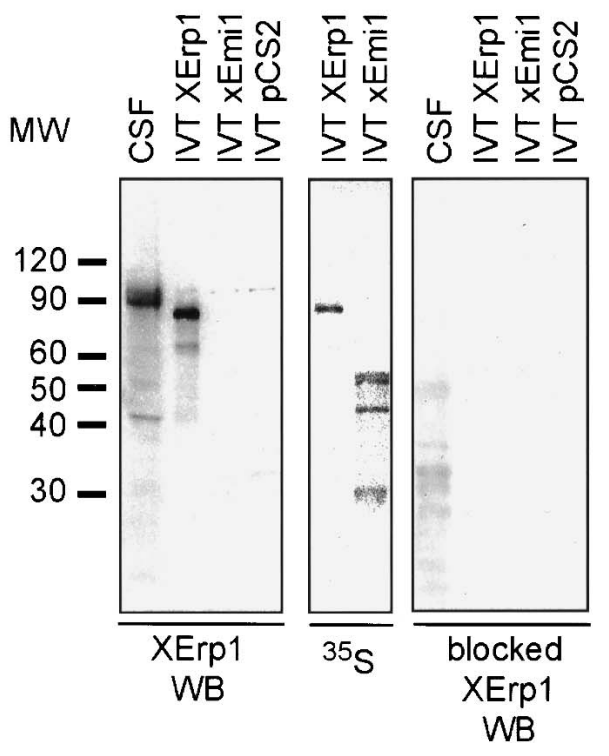

tract was incubated with the proteasome inhibitor MG132 before calcium addition, ${ }^{35}$ S-labeled IVT XErp1 was stabilized (data not shown), suggesting that the calciuminduced degradation of endogenous XErp1 is mediated by the ubiquitin-proteasome pathway. Once the released extract had entered interphase (40-60 min), XErp1 reappeared in a higher-mobility form (Fig. 2A, left panel), suggesting that XErp1 is phosphorylated in CSF but not in interphase extract. To test this we immunopurified ${ }^{35}$ S-labeled IVT XErp1 from CSF and interphase extract and treated the CSF form with phosphatase. Autoradiographic analysis revealed that phosphatase treatment converted the slower-migrating form of XErp1 into the higher-mobility form (Fig. 2B). These data indicate that the mobility difference between the interphase and $M$ phase forms of XErp1 reflects meiosis-specific phosphorylation of XErp1. In extract treated with the protein synthesis inhibitor cycloheximide, XErp1 was also degraded upon CSF release, but it did not reappear (Fig. 2A, right panel). Taken together, these data indicate that XErp1 is inactivated upon CSF release by proteasomal degradation and resynthesized in interphase extract.

\section{XErp1 accumulates during oocyte maturation}

Next, we wanted to know whether XErp1 accumulates during oocyte maturation. Oocyte maturation was induced by progesterone treatment of stage VI oocytes. At the indicated time points, equal numbers of oocytes were collected from a synchronous population and processed for immunoblot analysis (Fig. 2C). Immunoblot analyses for phosphorylated MAPK (Erk1/2), which is the active form of Erk1/2, and cyclin B levels (Fig. 2C) confirmed that the progesterone treatment induced oocyte maturation in our experiment. XErp1 was found to be expressed in stage VI oocytes, and its protein level increased significantly $4 \mathrm{~h}$ after progesterone treatment (Fig. 2C). Interestingly, XErp1 showed a shift in electrophoretic mobility $\sim 2.5 \mathrm{~h}$ after progesterone treatment, which remained until the oocytes entered meiosis II (Fig.

Figure 1. Domain structure of XErp1 and characterization of the XErp1 antibody. (A) The $\mathrm{N}$ terminus of XErp1 contains the putative $\mathrm{SCF}^{\beta-\mathrm{TRCP}}$ recognition sequence $\mathrm{DSGX}_{3} \mathrm{~S}$. In addition, XErpl protein contains a putative C-terminal F-box and a zincbinding region (ZBR). The C terminus of XErp1 shares 39\% sequence identity with Xenopus Emil. (B) ClustalW alignment of XErp1 protein sequences from different vertebrate species shows the evolutionary conservation of the XErp1 proteins. The amino acid sequences of XErp1 peptides that were identified by LC-MS/MS analysis of XErp1 are indicated (solid blue lines, peptide sequences in agreement with both XErp1 and Fbx26 ORF; dashed blue lines, peptide sequences in agreement only with XErp1 ORF). (C) Characterization of XErp1 antibodies. CSF extract or in vitro translated (IVT) XErp1, IVT xEmi1, or unprogrammed wheat germ extract were immunoblotted with affinity purified anti-XErp1 antibodies (left) or with affinity-purified anti-XErp1 antibodies blocked with XErp1 antigen (right). (Middle) The expression level of the ${ }^{35}$ S-labeled IVT proteins was examined by autoradiographic analysis. 
A

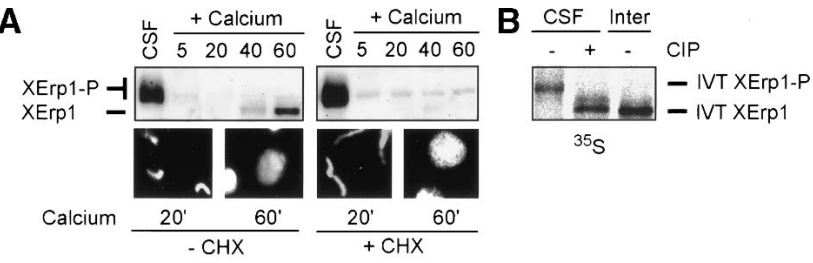

C

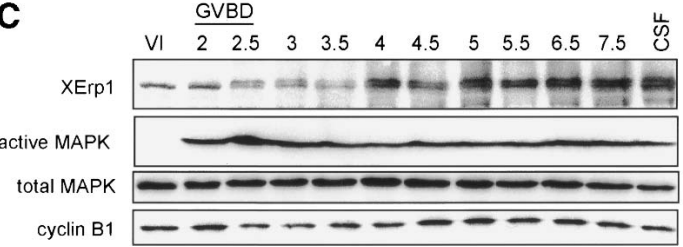

Figure 2. XErp1 accumulates during oocyte maturation and is destroyed upon CSF release. (A) CSF-arrested Xenopus egg extract was incubated at $20^{\circ} \mathrm{C}$ and treated with calcium to induce CSF release in the presence or absence of the translation inhibitor cycloheximide (CHX). At the indicated time points (minutes), samples were taken and immunoblotted for XErp1 protein. In addition, DNA morphology was observed to follow exit from CSF arrest. $(B)^{35}$ S-labeled IVT XErp1 was immunoprecipitated from CSF-arrested or interphase extract and treated with calf intestinal phosphatase (CIP). (C) Analysis of XErp1 protein levels during oocyte maturation. Stage VI oocytes where isolated and the maturation process induced by addition of progesterone. Oocytes were sampled at the indicated time points (minutes) after progesterone treatment and processed for immunoblot analysis of XErp1 and markers of the maturation process.

2C). The appearance of the upshifted XErp1 form coincided with a transient decrease in the level of cyclin B (Fig. 2C), which is characteristic for exit from meiosis I. Since we have shown that the differences in electrophoretic mobility between the $\mathrm{M}$-phase and interphasic forms of XErp1 are due to phosphorylation (Fig. 2B), it is likely that the observed upshift of XErp1 upon exit from meiosis I also reflects phosphorylation.

\section{XErp1 is essential for CSF arrest}

Since XErp1 is present in CSF extract, we asked whether XErpl is required to keep APC/C inactive in CSF extract. To test this, we added anti-XErp1 antibody immobilized on protein-G beads to CSF extract. Microscopic examination of the extract revealed that the chromatin decondensed even in the absence of calcium (Fig. 3A, panel 2), indicating that extract incubated with immobilized antiXErp1 antibodies prematurely entered interphase. In contrast, extract treated with immobilized control antibodies or anti-XErp1 antibodies preblocked with antigen maintained the CSF state, as indicated by condensed chromatin (Fig. 3A, panels 1,3). To confirm these results we next determined the cell cycle state of the differently treated extracts by measuring the activity of Cdk1. Cdk1 activity is high in CSF extract and decreases upon the degradation of cyclin B at anaphase onset. At the indicated time points, extract samples were taken and the activity of Cdk1 was analyzed using exogenous histone
$\mathrm{H} 1$ as substrate (Fig. 3A, right panel). Autoradiographic analysis revealed that Cdk1 activity remained high in extract treated with immobilized anti-XErp1 antibodies preblocked with antigen or control antibodies (Fig. 3A, right panel, lanes 1,3). In contrast, Cdk1 activity dropped in extract incubated with immobilized anti-XErp1 antibodies in the absence of calcium (Fig. 3A, right panel, lane 2), confirming that the addition of anti-XErp1 antibodies to CSF extract induces premature entry into interphase.

Next, we wanted to know whether the premature CSF release observed upon addition of immobilized antiXErp1 is specific to the inactivation of XErp1. Thus, we tested whether exogenous XErp1 could rescue the phenotype caused by XErp1 inactivation. Using an antibody raised against the $\mathrm{N}$ terminus of XErp1, we immunode-

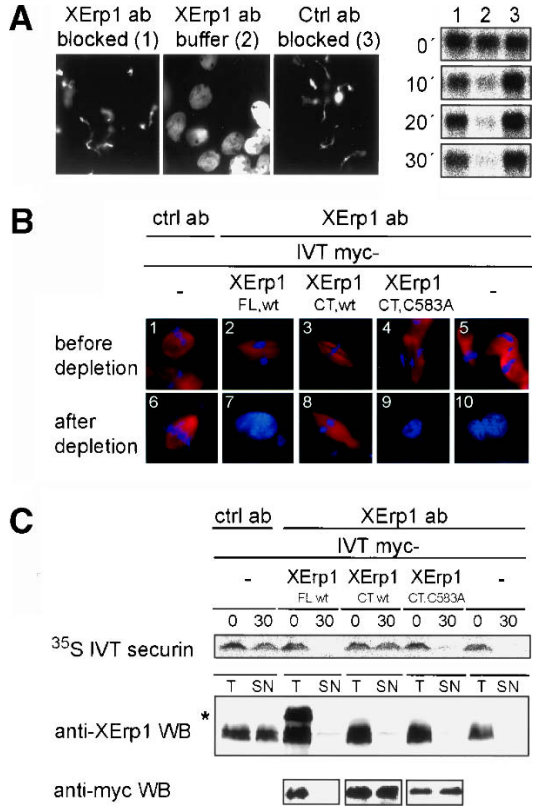

Figure 3. XErp1 is required for CSF activity. $(A)$ On ice, CSF extracts were treated with immobilized XErp1 antibody preincubated with a 10-fold molar excess of antigen (panel 1), buffer (panel 2), or control antibody preincubated with a 10-fold molar excess of antigen (panel 3). DNA morphology was examined 65 min after warming the extracts to $20^{\circ} \mathrm{C}$. Cdk1 activity was determined using an $\mathrm{H} 1$ kinase assay from samples withdrawn on ice $(0 \mathrm{~min})$ and 10,20 , and $30 \mathrm{~min}$ after warming the extracts to $20^{\circ} \mathrm{C}$. (B) CSF extracts were incubated for $20 \mathrm{~min}$ at $20^{\circ} \mathrm{C}$ with myc-tagged IVT XErp1 proteins and subsequently incubated with XErp1- or control-antibody beads on ice. Morphology of sperm nuclei and tubulin was examined before depletion and 55 min after warming the XErp1- and mock-depleted samples to $20^{\circ} \mathrm{C}$. (C) Total Xenopus extract (T) and the supernatant of depleted extract (SN) were immunoblotted for endogenous XErp1 (middle panel) and for myc-tagged IVT XErp1 proteins (lower panel). The asterisk $\left(^{\star}\right)$ indicates myc-tagged IVT XErp1 ${ }^{\mathrm{FL}, \mathrm{wt}}$ on the anti-XErp1 immunoblot. ${ }^{35}$ S-labeled IVT securin was detected by autoradiography. Samples were withdrawn before antibody addition (0) and $30 \mathrm{~min}$ after warming the extracts to $20^{\circ} \mathrm{C}(30)$. 
pleted XErp1 from CSF extract supplemented with either mock IVT or different myc-tagged IVT XErp1 proteins. Since the inactivation of XErp1 triggers the irreversible release of the extract from CSF arrest (Fig. 3A), the IVTs used for the rescue experiments had to be added to the extract before the immunodepletion of XErp1 was initiated. Immunoblot analysis confirmed that endogenous XErp1 was quantitatively removed from XErp1-depleted extracts but not from the extract treated with control antibodies (Fig. 3C, middle panel). As expected, IVT fulllength XErp1 was recognized by the anti-XErp1 antibody and therefore depleted from the extract along with the endogenous protein, but the two C-terminal fragments were not removed (Fig. 3C, lower panel). Microscopic examination revealed that control-depleted extract maintained the CSF state as indicated by condensed chromatin and bipolar spindle structures (Fig. 3B, panels $1,6)$. Consistently, IVT securin, a substrate of the APC/ C, remained stable in control-depleted extract (Fig. 3C, upper panel). As expected, XErp1-depleted extract supplemented with mock IVT could not maintain the CSF state but entered interphase prematurely, as indicated by decondensed chromatin and degraded IVT securin (Fig. 3B [panels 5,10], C [upper panel]). XErp1-depleted extract supplemented with full-length IVT XErp1 $\left(\mathrm{XErp} 1^{\mathrm{FL}, \mathrm{wt}}\right)$ also could not maintain the CSF state (Fig. $3 \mathrm{~B}$, panels 2,7) because the exogenous IVT XErp1, like the endogenous protein, was efficiently depleted from the extract (Fig. 3C, lower panel). In contrast, in extract supplemented with IVT XErp1 C terminus (XErp1 ${ }^{\mathrm{CT}, \mathrm{wt}}$ ), the chromatin stayed condensed, bipolar spindle structures remained, and IVT securin was not degraded (Fig. 3B [panels 3,8, C [upper panel]), demonstrating that IVT $\mathrm{XErp} 1^{\mathrm{CT}, \mathrm{wt}}$ could rescue the premature CSF release induced by depleting endogenous XErpl. However, a Cterminal fragment of XErp1 carrying a mutation in the ZBR (XErp1 ${ }^{\mathrm{CT}, \mathrm{C} 583 \mathrm{~A}}$ ) could not rescue the premature CSF release induced by depleting endogenous XErp1 (Fig. 3B [panels 4,9], C [upper panel]). Comparison of the signal intensities derived from endogenous XErp1 and IVT $\mathrm{XErp} 1^{\mathrm{FL}, \mathrm{wt}}$ (marked with an asterisk in Fig. 3C, antiXErp1 immunoblot) confirmed that the IVT product was present in the extract at a concentration similar to that of endogenous XErp1. The same applied to the different C-terminal XErp1 IVT products as judged from the antimyc immunoblot (Fig. 3C, lower panel). Taken together these data demonstrate that XErp1 is essential to keep the APC/C inactive in CSF extract, and that XErp1 requires a functional $\mathrm{ZBR}$ for its inhibitory effect on $\mathrm{APC} / \mathrm{C}$ activity.

\section{Excess XErp1 can prevent APC/C activation in CSF extract}

Given that the inactivation of XErp1 results in the premature activation of APC/C in Xenopus egg extract, we next asked whether excess XErp1 protein is sufficient to prevent CSF release. To test this we induced CSF release by adding calcium to Xenopus egg extract incubated with purified maltose-binding protein (MBP)-tagged
XErp1 $1^{\mathrm{FL}, \mathrm{wt}}$. Examination of DNA morphology revealed that the incubation of extract with $500 \mathrm{nM}$ MBP$\mathrm{XErp} 1^{\mathrm{FL}, \mathrm{wt}}$ prevented chromatin decondensation for $>60$ min (Fig. 4A), suggesting that the calcium-induced release of CSF extract had not taken place. Consistently, immunoblot analysis revealed that CDC27, a core APC/C subunit that shows a prominent upshift upon mitotic phosphorylation (Kotani et al. 1998), remained upshifted after calcium addition (Fig. 4C, upper panel). Moreover, the radiolabeled APC/C substrates IVT N-terminal fragment of cyclin $\mathrm{B} 1\left(\mathrm{cycB}^{\mathrm{NT}}\right)$ and securin were not degraded upon calcium addition in extract supplemented with MBP-XErp $1^{\mathrm{FL}, \mathrm{wt}}$ protein (Fig. 4C, middle and lower panels), indicating that the APC/C had not been activated. However, when CSF extract was supplemented with $500 \mathrm{nM}$ full-length XErp1 protein carrying a mutation in its ZBR (MBP-XErp $\left.1^{\mathrm{FL}, \mathrm{C} 583 \mathrm{~A}}\right)$, the chromatin decondensed (Fig. 4A), CDC27 lost its $M$ phase-specific upshift (Fig. 4C, upper panel), and the APC/C substrates IVT $\mathrm{cycB}^{\mathrm{NT}}$ and securin were degraded (Fig. 4C, middle and lower panels). These data suggest that excess XErp1 can prevent calcium-induced APC/C activation and that XErp1 requires a functional ZBR for its inhibitory effect on CSF release. This is in line with our previous observation that IVT XErp1 ${ }^{\mathrm{CT} \text {,wt }}$ but not IVT XErp $1^{\mathrm{CT}, \mathrm{C} 583 \mathrm{~A}}$ could rescue the premature CSF release induced by depletion of endogenous XErp1 (Fig. 3B,C). Notably, when we added a C-terminal fragment of XErp1 (MBP-XErp1 $\left.^{\mathrm{CT}, \mathrm{wt}}\right)$ at concentrations as low as $100 \mathrm{nM}$, the extract did not enter interphase for $>60 \mathrm{~min}$ after calcium addition, as judged from DNA morphology (Fig. 4B), whereas equimolar concentrations of the $N$ terminus of XErp1 (MBP-XErp1 $1^{\mathrm{NT}, \mathrm{wt}}$ ) or of MBP-XErp1 ${ }^{\mathrm{FL}, \mathrm{wt}}$ had no effect on CSF release (Fig. 4B). These data confirm that the $\mathrm{C}$ terminus of XErp1 is sufficient to prevent CSF release. In addition, the observation that the $\mathrm{C}$ terminus of XErpl is more potent in preventing CSF release than the full-length protein indicates that the $\mathrm{N}$ terminus of XErp1 exerts a negative regulatory influence on the activity of XErp1.

\section{MAPK pathway inhibition by UO126 does not prevent XErp1 action}

Next, we asked whether XErp1 is dependent on the Mos/ MAPK pathway. If this were the case, then the activity of the MAPK pathway should be essential for XErp1's inhibitory effect on CSF release. To test this we induced CSF release by adding calcium to Xenopus egg extract supplemented with MBP-XErp $1^{\mathrm{FL} \text {,wt }}$ in the presence of the MAPK kinase inhibitor UO126 or DMSO as a solvent control. To confirm that the MAPK pathway was inactivated upon UO126 addition, we performed immunoblot analyses for the active form of MAPK (Erk1/2). As shown in Figure 4E, active MAPK was present in Xenopus extract treated with DMSO but barely detectably in UO126-treated extract. Autoradiographic analyses revealed that ${ }^{35}$ S-labeled IVT securin remained stable upon calcium addition in the presence of MBP-XErp $1^{\mathrm{FL}, \mathrm{wt}}$ and UO126 (Fig. 4E), indicating that, under the conditions of 
A

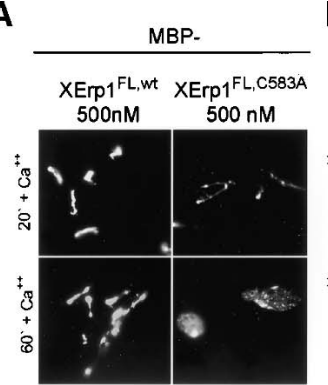

B

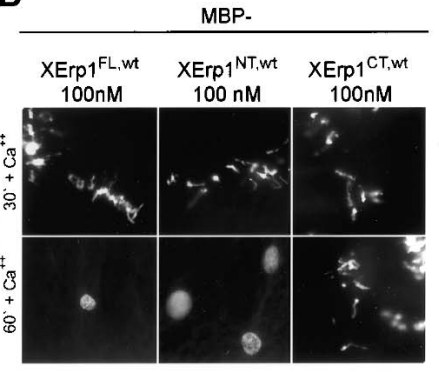

C

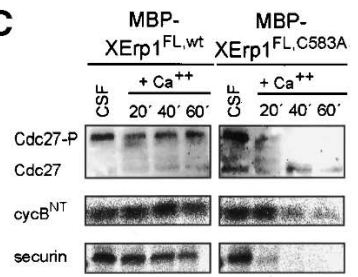

E
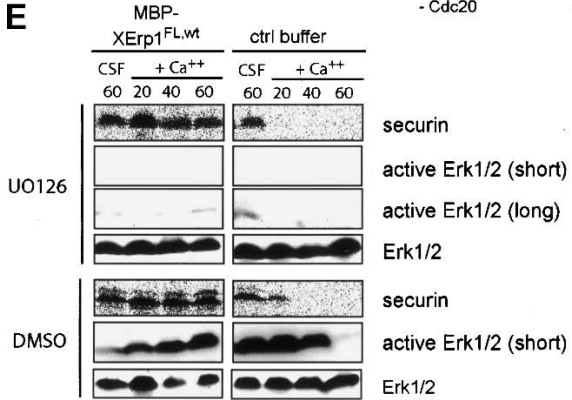

Figure 4. Excess XErp1 protein blocks calcium-dependent CSF release independently of Mos/MAPK signaling. (A). Purified MBP-tagged XErp $1^{\mathrm{FL} \text {,wt }}$ or XErp $1^{\mathrm{FL}, \mathrm{C} 583 \mathrm{~A}}$ was added to Xenopus egg extract at $500 \mathrm{nM}$ final concentration. Chromatin morphology was examined 20 and $60 \mathrm{~min}$ after calcium addition. (B) Purified MBP-tagged XErp1 $1^{\mathrm{FL}, \mathrm{wt}}$, XErp $1^{\mathrm{NT} \text {, wt }}$, or XErp1 $1^{\mathrm{CT}, \mathrm{wt}}$ were separately added to Xenopus egg extract at $100 \mathrm{nM}$ final concentration. Chromatin morphology was examined $30 \mathrm{~min}$ and $60 \mathrm{~min}$ after calcium addition. (C) Xenopus extract was treated as in $A$, and samples were taken at the indicated time points after calcium addition and immunoblotted for CDC27. Samples of extract supplemented with ${ }^{35}$ S-labeled IVT securin or N-terminal fragment of cyclin $\mathrm{B} 1\left(\mathrm{cycB}^{\mathrm{NT}}\right)$ were taken at the indicated time points before (CSF) and after calcium addition and analyzed by autoradiography. $(D)$ His-tagged Cdc20 was incubated with buffer (group 2), MBP-XErp1 ${ }^{\mathrm{CT}, \mathrm{wt}}$ (group 3, 300

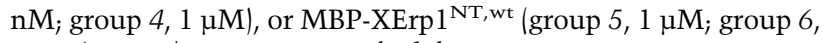
$3 \mu \mathrm{M})$. APC/C immunopurified from mitotic Xenopus extract was then mixed with buffer (group 1) or CDC20 incubated with buffer (group 2) or the indicated MBP-XErp1 fragments (groups $3-6)$ and assayed for its ability to ubiquitylate ${ }^{35}$ S-labeled IVT $\mathrm{cycB}^{\mathrm{NT}}$ at $20^{\circ} \mathrm{C}$. At the indicated time points, samples were taken and analyzed by autoradiography. (E) Purified MBP-tagged $\mathrm{XErp} 1^{\mathrm{FL}, \mathrm{wt}}$ or control buffer was added to CSF-arrested Xenopus egg extract in the presence or absence of the MAPK kinase inhibitor UO126. Inactivation of the MAPK pathway was confirmed by immunoblotting for active Erk1/2. The cell cycle state of the extract was monitored by the stability of exogenously added ${ }^{35}$ S-labeled IVT securin. the experiment, XErp1 does not depend on active MAPK for its ability to block CSF release. In extract supplemented with control buffer, ${ }^{35}$ S-labeled IVT securin was more rapidly degraded upon calcium addition in the presence of UO126 than in DMSO-treated extract, suggesting that the inactivation of the MAPK pathway can accelerate exit from meiosis II. Taken together, these data suggest that the ability of XErp1 to block CSF release does not strictly depend on the Mos/MAPK pathway.

\section{XErp1 can inhibit APC/C $C^{C D C 20}$ directly}

Given the sequence similarity between XErp1's C terminus and Emil (Fig. 1A), we asked whether the $\mathrm{C}$ terminus of XErp1, similar to Emil, could directly inhibit APC/C activity. To test this possibility we performed an in vitro ubiquitylation reaction using purified components, namely the ubiquitin-activating enzyme E1, the ubiquitin-conjugating enzyme $\mathrm{UbcX}$ as an E2, the $\mathrm{APC} / \mathrm{C}$ activator CDC20, APC/C immunopurified from mitotic Xenopus egg extract, and radiolabeled IVT cy$\mathrm{cB}^{\mathrm{NT}}$ as a substrate. In line with previous reports (Kramer et al. 1998), autoradiographic analyses revealed that the addition of CDC20 greatly enhanced APC/C's ubiquitin ligase activity towards its substrate $\mathrm{cycB}^{\mathrm{NT}}$ (Fig. $4 \mathrm{D}$, groups 1,2$)$. The addition of $300 \mathrm{nM} \mathrm{MBP-XErp1}{ }^{\mathrm{CT} \text {, wt }}$ strongly inhibited the $\mathrm{APC} / \mathrm{C}^{\mathrm{CDC} 2 \mathrm{O}}$-mediated ubiquitylation of IVT $\mathrm{cycB}^{\mathrm{NT}}$, whereas the addition of $1 \mu \mathrm{M}$ of MBP-XErp1 ${ }^{\mathrm{CT} \text {,wt }}$ almost completely abolished the CDC20-dependent ubiquitylation reaction (Fig. 4D, groups 3,4$)$. In contrast, addition of $1 \mu \mathrm{M}$ or $3 \mu \mathrm{M}$ of MBPXErp1 $1^{\mathrm{NT} \text {,wt }}$ had no significant effect on the Cdc20-dependent ubiquitylation of IVT cyclin B1 N terminus (Fig. $4 \mathrm{D}$, groups 5,6 ). These data strongly support our model that XErp1 is an APC/C inhibitor and that the C terminus of XErp1 accounts for the inhibitory effect of XErp1 on CSF release by directly inhibiting $\mathrm{APC} / \mathrm{C}^{\mathrm{CDC} 20}$. So far, we have not been able to convincingly demonstrate a direct interaction between XErp1 and Cdc20, although such an interaction has been reported for Emil and Cdc20 (Reimann et al. 2001b; Reimann and Jackson 2002). Thus the precise mechanism of the inhibition of $\mathrm{APC} / \mathrm{C}^{\mathrm{Cdc} 20}$ is presently unknown.

\section{Plx1 phosphorylates XErp1 in vitro}

Because XErp1 was initially identified as a Plx1-interacting protein, we next sought to explore the functional relationship between XErp1 and Plx1 in the regulation of CSF arrest and APC/C activity upon M-phase exit in Xenopus egg extract. First, we tested whether XErp1 is a substrate of Plx1 in vitro. Using MBP-fusion proteins we could show that recombinant Plxl efficiently phosphorylates full-length XErp1 and an N-terminal fragment of XErp1 in vitro (Fig. 5A). In contrast, XErp $1^{\mathrm{CT} \text {,wt }}$ containing the C-terminal F-box and the ZBR was not detectably phosphorylated (Fig. 5A). The fact that Plx1 efficiently phosphorylated XErp $1^{\mathrm{FL} \text {, wt }}$ and XErp $1^{\mathrm{NT} \text {, wt }}$ but not equimolar concentrations of XErp1 ${ }^{\mathrm{CT} \text {,wt }}$ (Fig. 5A) 
Schmidt et al.
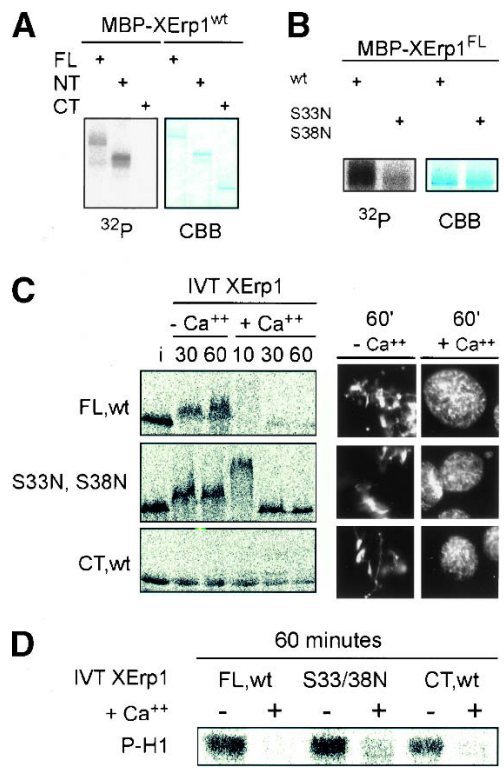

Figure 5. Plx1-dependent destabilization of XErp1 is required for CSF release. (A) MBP-tagged $\mathrm{XErp} 1^{\mathrm{FL}, \mathrm{wt}}, \mathrm{XErp} 1^{\mathrm{NT}, \mathrm{wt}}$, and $\mathrm{XErp} 1^{\mathrm{CT}, \mathrm{wt}}$ were each incubated in an in vitro phosphorylation reaction with His-tagged $\mathrm{Plx} 1$. Incorporation of ${ }^{32} \mathrm{P}$ was analyzed by PAGE and autoradiography. $(B)$ In vitro Plx1 kinase assay as in $A$ using MBP-tagged wild-type XErp1 $1^{\mathrm{FL}, \mathrm{wt}}$ or $\mathrm{XErp1}^{\mathrm{FL}, \mathrm{S} 33 \mathrm{~N}, \mathrm{~S} 38 \mathrm{~N}}$. (C) ${ }^{35}$ S-labeled IVT XErp $1^{\mathrm{FL}, \mathrm{wt}}, \mathrm{XErp} 1^{\mathrm{CT} \text {, wt }}$, or a mutant form of $\mathrm{XErp} 1$ (XErp $1^{\mathrm{FL}, \mathrm{S} 33 \mathrm{~N}, \mathrm{~S} 38 \mathrm{~N}}$ ) were incubated in CSF extract (i, input) in the presence or absence of calcium. At the indicated time points, samples were withdrawn and analyzed by autoradiography. (D) Cdk1 activity of all samples shown in $C$ withdrawn after 60 min was measured using an $\mathrm{H} 1$ kinase assay.

indicates that the conditions of the in vitro assay did not promote unspecific protein phosphorylation by Plxl. These data support our hypothesis that the activity of XErp1 is regulated by Plx1-dependent phosphorylation of XErp1's $\mathrm{N}$ terminus. In line with these results, we observed that Plx1 showed a yeast-two-hybrid interaction with the $\mathrm{N}$ terminus but not the $\mathrm{C}$ terminus of XErp1 (data not shown).

\section{XErp1 is targeted for degradation via its} $D S G X_{3} S$ motif

The $\mathrm{N}$ terminus of XErp1 contains a DSGX $\mathrm{S}_{3}$ motif (Fig. $1 \mathrm{~A}, \mathrm{~B})$. It has been shown previously that the phosphorylation of the two serine residues of a $\mathrm{DSGX}_{2 / 3} \mathrm{~S}$ motif targets substrates for degradation by the SCF (Skp1-Cullin F-box) ubiquitin ligase complex that contains the F-box protein $\beta$-TRCP (SCF ${ }^{\beta-T R C P}$ ) (Fuchs et al. 2004). We thus asked whether the degradation of XErp1 upon CSF release is mediated by its DSGX $_{3} S$ motif. To test this possibility we added ${ }^{35}$ S-labeled IVT XErp1 to Xenopus egg extract and studied the stability of IVT XErp1 upon calcium addition. For these experiments the different IVT XErp1 proteins were added to the CSF extract at a concentration low enough (one-fourth of the amount used for the add-back experiment, Fig. 3C) to assure that the exogenous XErp1 did not block CSF release. IVT $\mathrm{XErp} 1^{\mathrm{FL}, \mathrm{wt}}$, like the endogenous protein, was stable in CSF extract but was rapidly degraded upon calcium addition (Fig. 5C). In contrast, neither an IVT C-terminal XErp1 fragment (amino acids 374-651) lacking the DSGX $_{3} \mathrm{~S}$ motif nor IVT full-length XErp1 protein mutated at Ser 33 and Ser 38 of the DSGX $_{3}$ S motif $\left(\mathrm{XErp} 1^{\mathrm{S} 33 \mathrm{~N}, \mathrm{~S} 38 \mathrm{~N}}\right)$ were degraded upon calcium addition (Fig. 5C). Examination of DNA morphology (Fig. 5C, right panel) and of Cdk1 kinase activity (Fig. 5D) confirmed that extracts incubated with the different IVT XErp1 products entered interphase upon calcium addition. Intriguingly, IVT full-length XErp1 mutated in its $\mathrm{DSGX}_{3} \mathrm{~S}$ motif showed a transient hypershift upon calcium addition (Fig. 5C, 10 min after calcium [or $\mathrm{Ca}^{++}$] addition). This hypershift seems to be caused by phosphorylation, because the slower-migrating form observed $10 \mathrm{~min}$ after calcium addition remained upshifted when the extract was diluted into buffer containing the phosphatase inhibitor okadaic acid but not when the extract was diluted into buffer only (data not shown). The question of whether Plx1 contributes to this calciumdependent hypershift will be addressed below. These data show that the $\mathrm{DSGX}_{3} \mathrm{~S}$ sequence targets XErp1 for degradation in a cell cycle-dependent manner.

Next we wanted to know whether Plxl could contribute to the degradation of XErp1 by directly phosphorylating the serine residues of the $\mathrm{DSGX}_{3} \mathrm{~S}$ motif. We thus performed additional in vitro Plx1 kinase assays using MBP-tagged $\mathrm{XErp} 1^{\mathrm{FL}, \mathrm{wt}}$ and $\mathrm{XErp} 1^{\mathrm{S} 33 \mathrm{~N}, \mathrm{~S} 38 \mathrm{~N}}$ as substrates. As shown in Figure $5 \mathrm{~B}$, the phosphorylation of $\mathrm{XErp} 1^{\mathrm{S} 33 \mathrm{~N}, \mathrm{~S} 38 \mathrm{~N}}$ was significantly reduced compared to the wild-type protein, indicating that serine residues 33 and/or 38 of XErp1 are major Plx1 phosphorylation sites in vitro. Again, the fact that XErpl carrying two point mutations was a very poor Plxl substrate compared to equimolar concentrations of the wild-type protein supports the specificity of the observed phosphorylation. Taken together, these data suggest that Plx1 phosphorylates XErp1 at its DSGX $\mathrm{D}_{3}$ motif, which then serves as a "phospho-degron" (Fuchs et al. 2004) to target XErp1 for degradation upon CSF release. These data are consistent with recent reports showing that Plk1 can phosphorylate the $\mathrm{DSGX}_{2} \mathrm{~S}$ motif of Emil, thereby promoting its in vitro ubiquitylation (Hansen et al. 2004; Moshe et al. 2004). Thus, polo-like kinases are involved in the regulated degradation of both Emil and XErp1.

\section{Dominant-negative polo-box domain of Plx1 (PBD $\left.{ }^{w t}\right)$ stabilizes XErp1}

Previously, it has been shown that the addition of kinase-dead Plx1 (Plx1 $\left.{ }^{\mathrm{N} 172 \mathrm{~A}}\right)$ to Xenopus egg extract prevents APC/C activation and CSF release (Descombes and Nigg 1998). A plausible model for the inhibitory effect of $\mathrm{Plx} 1^{\mathrm{N} 172 \mathrm{~A}}$ on CSF release is that the $\mathrm{APC} / \mathrm{C}$ inhibitor $\mathrm{XErp} 1$ is stabilized through sequestration by excess catalytically inactive $\mathrm{Plx} 1^{\mathrm{N} 172 \mathrm{~A}}$. If $\mathrm{Plx} 1^{\mathrm{N} 172 \mathrm{~A}}$ sequesters potential substrates via its polo-box domain (PBD), then the addition of PBD alone should be sufficient to exert a 
dominant-negative effect on CSF release. Indeed, addition of recombinant MBP-tagged wild-type polo-box domain of Plx1 $\left(\mathrm{PBD}^{\mathrm{wt}}\right)$ inhibited DNA decondensation, spindle disassembly, and the degradation of IVT securin upon calcium treatment (Fig. 6A). In contrast, the addition of equimolar amounts of mutant PBD (Elia et al. 2003) (MBP-PBD ${ }^{\text {mut}}$ ) had no effect on CSF release, as judged by the appearance of nuclei and the degradation of IVT securin upon calcium addition (Fig. 6A). In line with these results, it was recently reported that a point mutation in the PBD of kinase-dead Plx1 (Plx $\left.1^{\mathrm{N} 172 \mathrm{~A}, \mathrm{~W} 408 \mathrm{~F}}\right)$ neutralizes the inhibitory effect of $\mathrm{Plx} 1^{\mathrm{N} 172 \mathrm{~A}}$ on $\mathrm{APC} / \mathrm{C}$ activation (Liu et al. 2004).

Next, we tested whether ${ }^{35}$ S-labeled IVT XErp1 is degraded upon calcium addition when CSF extract was preincubated with MBP-PBD ${ }^{\mathrm{wt}}$. As shown in Figure 6B, IVT $\mathrm{XErp} 1^{\mathrm{FL}}{ }^{\mathrm{wt}}$ remained stable upon calcium addition in the presence of MBP-PBD ${ }^{\mathrm{wt}}$ (compare 60-min time point before and after calcium addition). Since ${ }^{35}$ S-labeled IVT $\mathrm{XErp} 1^{\mathrm{FL}, \mathrm{wt}}$ became at least partially upshifted in CSF extract supplemented with MBP-PBD ${ }^{\mathrm{wt}}$ (Fig. 6B, cf. input [i] and the 30-min time point in the absence of calcium), it seems likely that the meiosis-specific phosphorylation of XErp1 does not completely depend on active Plx1. The identity of other kinases acting upon XErp1 is not presently known, but Cdk1 is a likely candidate. In contrast to the situation where the extract was supplemented with dominant-negative MBP-PBD, IVT XErp $1^{\mathrm{FL} \text {,wt }}$ was rapidly degraded after calcium addition in the buffer control (Fig. 6B). As expected, IVT XErp1 $1^{\mathrm{S} 33 \mathrm{~N}, \mathrm{~S} 38 \mathrm{~N}}$ remained stable upon calcium addition in the presence of MBP$\mathrm{PBD}^{\mathrm{wt}}$ or in the buffer control (Fig. 6B). Interestingly, the previously observed calcium-induced hypershift (see Fig.
5C) could only be observed in the buffer control, but not in extract supplemented with MBP-PBD ${ }^{\text {wt }}$ (Fig. 6B). This hypershift seems to be caused by phosphorylation (see above), suggesting that Plxl activity is required-directly or indirectly-for the phosphorylation of endogenous XErp1 upon calcium addition at a site different from the $\mathrm{DSGX}_{3} S$ motif. Perhaps this additional calcium-induced phosphorylation event negatively regulates the activity of XErp1 before XErp1 is targeted for degradation by the phosphorylation of its $\operatorname{DSGX}_{3} S$. Taken together, these data suggest that that active Plx1 is required for the degradation of XErp1 via phosphorylation of its $\mathrm{DSGX}_{3} \mathrm{~S}$ motif at anaphase onset, and that degradation of XErp1 is a prerequisite for CSF release.

\section{XErp1 is required for the PBD ${ }^{w t}$-induced block of CSF release}

Thus far our data have shown that XErp1 is essential for CSF arrest and that the activity of Plx1 is required to mediate the degradation of XErp1 upon CSF release. If $\mathrm{XErp} 1$ is the critical downstream effector of $\mathrm{Plx} 1$ in regulating the APC/C, one would predict that the activity of XErp1 is crucial for the dominant-negative effect of the $\mathrm{PBD}^{\mathrm{wt}}$ on CSF release. To test this we examined whether $\mathrm{PBD}^{\mathrm{wt}}$ can still block CSF release under conditions where XErp1 is kept inactive by the addition of immobilized anti-XErp1 antibodies. Since the inactivation of XErp1 induces a premature calcium-independent CSF release (Fig. 3), it was important to add the antibodies to extract already incubated with $\mathrm{PBD}^{\mathrm{wt}}$. When we added immobilized anti-XErp1 antibodies to extract supplemented with MBP-PBD ${ }^{\mathrm{wt}}$, the chromatin was decon-
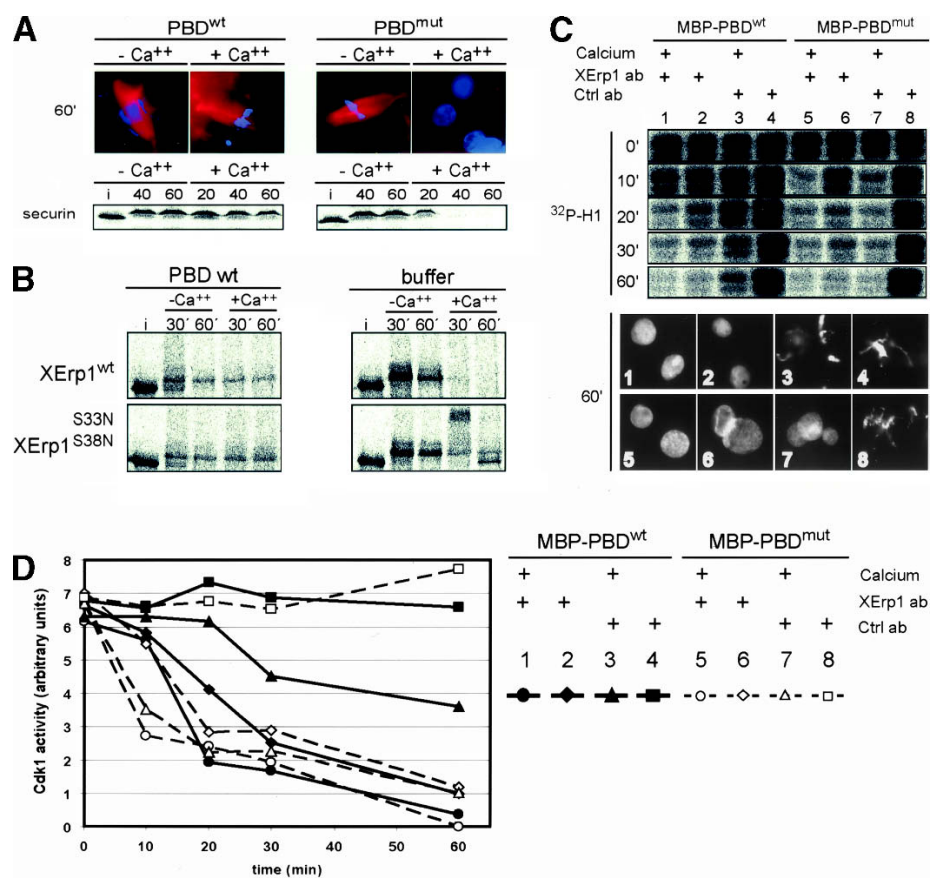

Figure 6. XErp1 is required for effects of dominantnegative PBD on CSF release $(A)$ CSF extracts were incubated with equimolar amounts of MBP-tagged either wild-type $\left(\mathrm{PBD}^{\mathrm{wt}}\right)$ or mutant $\mathrm{PBD}\left(\mathrm{PBD}^{\mathrm{mut}}\right)$ of $\mathrm{Plx} 1$. Morphology of DNA and tubulin was examined after extracts had been warmed to $20^{\circ} \mathrm{C}$ for $60 \mathrm{~min}$ in the presence or absence of calcium. In addition, the stability of exogenously added ${ }^{35} \mathrm{~S}$-labeled IVT securin was monitored by autoradiography. $(B){ }^{35}$ S-labeled IVT $\mathrm{XErp} 1^{\mathrm{FL}, \mathrm{wt}}$ or XErp $1^{\mathrm{FL}, \mathrm{S} 33 \mathrm{~N}, \mathrm{~S} 38 \mathrm{~N}}$ were incubated in CSF extract containing $\mathrm{PBD}^{\mathrm{wt}}$ or control buffer. Extracts were warmed to $20^{\circ} \mathrm{C}$, and samples were taken and examined by autoradiography at the indicated time points before and after addition of calcium. $(C)$ Immobilized anti-XErp1 antibodies (lanes 1,2,5,6) or immobilized control antibodies (lanes $3,4,7,8$ ) were added to CSF extract containing $\mathrm{PBD}^{\mathrm{WT}}$ (lanes $1-4$ ) or $\mathrm{PBD}^{\text {mut }}$ (lanes $5-8$ ) in the presence (lanes 1,3,5,7) or absence (lanes $2,4,6,8)$ of calcium. Samples were taken at the indicated time points after calcium and/or antibody addition and assayed for Cdk1 activity. In addition, samples of the reactions (panels 1-8) were withdrawn and chromatin morphology was examined $65 \mathrm{~min}$ after warming the extracts to $20^{\circ} \mathrm{C}$. (D) Resulting bands of the $\mathrm{Hl}$ kinase assay were quantified by densitometry. Band intensities were corrected for background and then plotted against time. Solid lines show reactions with $\mathrm{PBD}^{\mathrm{WT}}$; dashed lines show reactions with $\mathrm{PBD}^{\mathrm{mut}}$. 
densed 60 min after calcium addition (Fig. 6C, panel 1) and Cdk1 activity rapidly dropped to interphase levels (Fig. 6C [lane 1], D). In contrast, the chromatin remained condensed when calcium was added to extract supplemented with MBP-PBD ${ }^{\text {wt }}$ and immobilized control antibodies (Fig. 6C, panel 3). Under these conditions Cdk1 activity decreased by only $\sim 40 \%$, consistent with a block in CSF release (Fig. 6C [lane 3], D). These data confirm our model that $\mathrm{PBD}^{\mathrm{wt}}$ requires XErp1 to mediate its inhibitory effect on CSF release.

Xenopus egg extract incubated with MBP-PBD ${ }^{\text {wt }}$ and immobilized anti-XErp1 antibodies had low Cdk1 activity levels and decondensed chromatin even in the absence of calcium (Fig. 6C [lane 2], D [panel 2]), indicating that $\mathrm{PBD}^{\mathrm{wt}}$ cannot prevent the premature CSF release induced by inactivation of XErp1. As expected, Xenopus extract supplemented with MBP-PBD ${ }^{\text {mut }}$ entered interphase only in the presence of calcium or upon addition of anti-XErp1 antibodies, as indicated by low Cdk1 activity and decondensed chromatin (Fig. 6C [lanes 5-7], D [panels 5-7]). Taken together these observations show that XErp1 is required for the dominant-negative effect of $\mathrm{PBD}^{\mathrm{wt}}$ on CSF release and suggest that XErp1 acts downstream of Plx1. They are also consistent with our hypothesis that XErpl is a direct substrate of Plx1, implicated in the regulation of the APC/C ubiquitin ligase.

\section{Discussion}

Multiple independent pathways contribute to CSF activity in Xenopus eggs (Fig. 7). Here, we characterize XErp1, a novel evolutionarily conserved protein essential for CSF arrest in Xenopus egg extract. Excess XErp1 can prevent calcium-induced CSF release, and the $C$ terminus of XErp1 containing the ZBR is necessary and sufficient to prevent CSF release. Consistently, the $\mathrm{C}$ terminus of XErp1 inhibits the ubiquitin ligase activity of APC/ $\mathrm{C}^{\mathrm{CDC} 20}$ directly. In addition to the ZBR, XErpl contains an F-box at its $\mathrm{C}$ terminus. Yeast two-hybrid analyses revealed that the F-box of XErp1 is required for its interaction with the F-box-binding protein Skp1 (P.I. Duncan and E.A. Nigg, unpubl.), making XErp1 a bona fide F-box protein. However, the F-box does not seem to contribute to XErp1's inhibitory effect on APC/C activation, because IVT full-length XErp1 mutated in its F-box XErp $1^{\mathrm{L} 450 \mathrm{~A}}$ was equally potent as IVT XErp $1^{\text {WT }}$ in blocking calcium-induced CSF release (A. Schmidt, N.R. Rauh, and T.U. Mayer, unpubl.). Similarly, the F-box of Emil also seems to be dispensable for Emil's ability to inhibit APC/C (Reimann et al. 2001a). Therefore, the elucidation of the functional role of the F-Box of XErp1 and Emil will require further research.

The question arises of why Emil and XErp1, both of which are able to inhibit APC/C activity directly, seem to be essential to maintain the CSF arrest in Xenopus egg extract. Our data and previous reports have shown that extract depleted of one of these proteins cannot maintain the CSF arrest, although the remaining APC/C inhibiting protein should still be present in the extract. One could imagine that the premature CSF release observed here is actually due to Emil depletion or Emil codepletion with XErp1. However, three independent lines of evidence argue against this idea. First, the antibody used for immunodepletion did not detect IVT Emil in immunoblot analyses (Fig. 1C). Second, we have observed no indication of an interaction between XErp1 and Emil, since mass spectrometry analyses of proteins associated with endogenous XErp1 did not identify Emil (G. Sauer and T.U. Mayer, unpubl.). Third and most importantly, decondensation of the sperm DNA, disassembly of the spindles, and degradation of securin upon XErp1-depletion could be rescued by the addition of IVT XErp $1^{\mathrm{CT}, \mathrm{wt}}$ at a concentration matching that of endogenous XErp1. These data clearly demonstrate that the observed premature APC/C activation results from the loss of XErp1 protein.

It is possible that XErp1 and Emil are part of parallel pathways that cooperate to regulate APC/C activity in Xenopus egg extract. If so, depleting one of these two proteins could lower the APC/C inhibitory activity in the extract below a critical concentration, resulting in premature APC/C activation. Similarly, the depletion of Cdk2 protein by an antisense approach abolishes CSF arrest in Xenopus eggs despite the presence of an active c-Mos/MAPK/p90 Rsk pathway, Emi1, and XErp1 (Gabrielli et al. 1993). These data suggest that the combined activities of the c-Mos/MAPK/p90 Rsk pathway, Cdk2/ cyclin E, Emil, and XErp1 are required to assure complete APC/C inhibition during CSF arrest. Consistently, the APC/C substrate cyclin B is completely stabilized during CSF arrest but degraded to $\sim 50 \%$ of the metaphase level during the meiosis I-to-II transition (Tunquist and Maller 2003), a time when Cdk2/cyclin E is absent. Interestingly, XErp1 is present upon exit from meiosis I, suggesting that the $\beta$-TRCP-mediated degradation of XErp1 is not operating at this early stage of meiotic progression. However, XErp1 apparently does

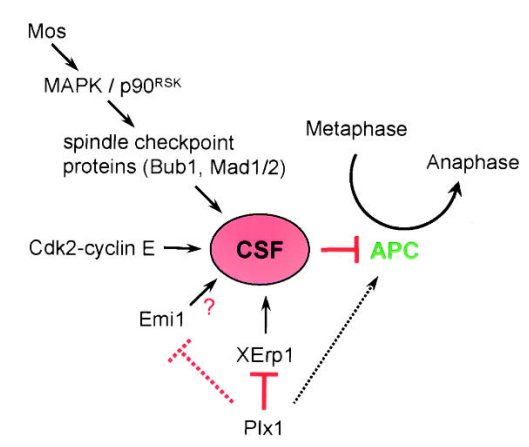

Figure 7. Model of different pathways regulating CSF activity. The c-Mos/MAPK/p90 Rsk pathway seems to exert its inhibitory effect on APC/C by activating components of the spindle assembly checkpoint. The underlying mechanism by which Cdk2/cyclin E inhibits APC/C activity has not been identified. XErp1 and Emil mediate CSF activity by inhibiting APC/C directly. Plx1 targets XErp1 and possibly Emil for degradation, thereby allowing APC/C activation. Additionally, Plx1 might activate APC/C directly by phosphorylating specific APC/C subunits. 
not block APC/C during meiosis I. Whether this is due to insufficient XErp1 levels or its functional inactivation (e.g., by the post-translational modification shown in Fig. 2C) remains to be explored.

In a recent publication, the role of Emil during CSF arrest in Xenopus egg extracts was challenged (Ohsumi et al. 2004). In particular, Kishimoto and coworkers (Ohsumi et al. 2004) reported that wild-type Emil is rapidly degraded during oocyte maturation and highly unstable in CSF extracts, raising the question of whether this protein could be present at sufficient levels in CSF-arrested extracts to perform its alleged role as an APC/C inhibitor (Reimann et al. 2001b; Reimann and Jackson 2002). Moreover, contradictory data exist concerning the fate of Emil upon calcium-induced CSF release. Whereas Emil was originally reported to be stable upon CSF release until the first mitotic division (Reimann et al. 2001b; Reimann and Jackson 2002), a more recent study from the same laboratory proposes that Plx1 triggers Emil destruction (Hansen et al. 2004), similar to the results reported here for XErp1. Clearly, further studies will be required to clarify the precise role of Emil during CSF arrest and release.

Irrespective of the controversy around the role of Emil in regulating CSF release, our present data strengthen the conclusion that the activity of Plxl is required to activate APC/C upon CSF release (Descombes and Nigg 1998) and to maintain APC/C in its active form (Brassac et al. 2000). Since polo-like kinases can phosphorylate specific APC/C subunits in vitro, the model emerged that polo-like kinases can activate the APC/C directly. However, it is still controversial whether APC/C phosphorylation by polo-like kinases is sufficient (Kotani et al. 1998), required (Golan et al. 2002), or even dispensable for the activation of the APC/C (Kraft et al. 2003). Our present data lead to a model in which Plx1 controls APC/C activity at anaphase onset by targeting XErp1, an inhibitor of $\mathrm{APC} / \mathrm{C}$, for degradation. According to this model, the inhibition of CSF release observed upon addition of catalytically inactive $\mathrm{Plx} 1^{\mathrm{N} 172 \mathrm{~A}}$ to Xenopus egg extract is mediated by the sequestration of XErp1 by the polo-box domain of $\mathrm{Plx}^{\mathrm{N} 172 \mathrm{~A}}$. Consistently, our data confirm that the polo-box domain of Plx1 requires XErp1 to exert its inhibitory effect on CSF release in Xenopus egg extract. The phosphorylation of XErp1, like that of other F-box proteins, seems to be a prerequisite for its degradation by the ubiquitin-proteasome pathway. Our data show that Plxl can phosphorylate a DSGX $\mathrm{D}_{3} \mathrm{~S}$ motif located within the $\mathrm{N}$ terminus of XErp1, and that the DSGX $_{3} \mathrm{~S}$ motif is essential for the degradation of XErp1 upon CSF release. Previously, it has been shown for other proteins that the phosphorylation of both serine residues of a $\mathrm{DSGX}_{2 / 3} \mathrm{~S}$ motif creates a "phospho-degron" recognized by the $\mathrm{SCF}^{\beta-\mathrm{TRCP}}$ ubiquitin ligase complex (Fuchs et al. 2004). Therefore, it appears plausible that $\mathrm{SCF}^{\beta-T R C P}$ also mediates the degradation of phosphorylated XErp1 upon CSF release.

The role of fertilization and the resulting calcium signal in controlling the functional interaction between Plx1, XErp1, Emil, and APC/C remain to be determined in future studies. The regulation of XErp1 function and stability is undoubtedly complex. As indicated by the several distinct electrophoretic mobilities associated with XErp1, this protein undergoes multiple post-translational modifications, including phosphorylation not only within the $\mathrm{DSGX}_{3} \mathrm{~S}$ degron, but also on other sites. The available evidence indicates that XErp1 is stable and active as an APC/C inhibitor in CSF extracts, although Plxl can be recovered from such extracts in an active form. Thus, the Plx1-dependent inactivation of XErp1 clearly depends on a calcium signal. It is possible that calcium sensitizes XErp1 to additional phosphorylation by Plx1 (e.g., through the action of a calcium-activated kinase). Alternatively, it would seem premature to rigorously exclude that the $\beta$-TRCP system could be regulated by calcium. Regardless of these open questions, our present data clearly indicate that APC/C activation by polo-like kinase in Xenopus egg extract involves not only direct phosphorylation of APC/C subunits but also the derepression of APC/C by inactivation of inhibitory proteins.

\section{Materials and methods}

\section{Yeast two-hybrid screen}

A yeast two-hybrid screen was performed as described previously (Gietz and Woods 2002). Bait plasmid pGBDU/Plx1 $1^{\mathrm{N} 172 \mathrm{~A}}$ was transformed into yeast strain PJ69-4A and selected by uracil auxotrophy. A Xenopus laevis oocyte matchmaker cDNA library in pACT2 (Clontech) was subsequently transformed into yeast strain PJ69-4A carrying the bait plasmid pGBDU/ $\mathrm{Plx}^{\mathrm{N} 172 \mathrm{~A}}$. Transformants were selected for growth on plates lacking uracil, leucine, and adenine. pACT2 plasmids were rescued by transforming HB101 bacteria with yeast DNA preparations, followed by selection for leucine auxotrophy. Plasmid DNA from positive clones was retransformed, and the cDNA inserts of positive clones were sequenced to identify putative Plx1-interacting proteins.

\section{Cloning and protein expression}

Full-length XErp1 (XErp1 $\left.{ }^{\mathrm{FL}, \mathrm{wt}}\right)$ was initially subcloned from the identified two-hybrid plasmid into a modified pCS2 vector introducing restriction sites for the FseI and AscI restriction enzymes. Primers with the sequences $5^{\prime}$-ATTATGGCCGGC CAGAGATGGCAAATCTCTTAGAG- $3^{\prime}$ and $5^{\prime}$-ATTATGGC GCGCCGGAAGACTAGCTTCAAAGTCTC-3' were used to amplify the coding sequence (start and stop codons are underlined). Site-directed mutagenesis to yield the C583A mutation in the ZBR was carried out using the QuikChange kit (Stratagene) according to the manufacturer's instructions. The $\mathrm{N}$-terminal and C-terminal fragments of XErp1 comprise the amino acids 1-424 and 374-651, respectively. Wild-type or mutant fragments were subcloned from plasmids containing the desired full-length XErp1 as a template following the strategy described above. The polo-box domain construct $\left(\mathrm{PBD}^{\mathrm{wt}}\right)$ comprising amino acids $358-598$ of $\mathrm{Plx} 1$ was subcloned from full-length Plx1 in a pCS2-Myc plasmid using primers 5'-ATTATGGCC GGCCGGAGTTCACG GAGCCTGC-3' and 5'-ATTATGGC GCGCCCTATGCCGAGGCCTTTAC-3'. A W408F, H532A, $\mathrm{K} 534 \mathrm{~A}$ mutation in polo box 1 ( $\mathrm{PBD}^{\mathrm{mut}}$ ) was introduced as described previously (Liu et al. 2004). Wild-type, mutant, and frag- 
ments of XErp1 protein as well as the polo-box domain of Plx1 were expressed from a modified pMal-vector (New England Biolabs) as an N-terminal MBP-fusion. The purifications were performed according to the manufacturer's instructions. Purification of his-tagged Plx1 from Sf9 cells was performed as described (Descombes and Nigg 1998).

\section{Antibody production}

6xHis-tagged N-terminal fragment of XErp1 (amino acids 105374) was expressed from a pQE31 plasmid in Escherichia coli strain BL21 (DE3)pLysS, purified under denaturing conditions with $\mathrm{Ni}^{2+} \mathrm{NTA}$ resins as recommended by the manufacturer (QIAGEN), and subsequently gel-purified after SDS-PAGE. Purified protein was used to immunize New Zealand white rabbits (Elevage Scientifique des Dombes). Antibodies were affinity-purified following standard procedures.

\section{CSF extracts}

Xenopus CSF egg extracts were prepared essentially as described previously (Murray 1991). CSF release was induced by adding $600 \mu \mathrm{M} \mathrm{CaCl}{ }_{2}$ to the extract. DNA and spindle morphology were examined as described previously (Desai et al. 1999). All experiments were carried out with freshly prepared extracts.

\section{Mass spectrometry}

Coomassie-stained protein bands were in-gel digested by trypsin, desalted, and concentrated using C18 extraction tips as reported (Shevchenko et al. 1996; Rappsilber et al. 2003). The peptide mixture was eluted from a pulled fused silica capillary with an internal diameter of $75 \mu \mathrm{m}$ and a tip opening of $8 \mu \mathrm{m}$ (New Objective) filled with ReproSil-Pur C18-AQ (Dr. Maisch, $\mathrm{GmbH}$, Ammerbuch, Germany) 3-mm reverse-phase material directly into a quadrupole time-of-flight mass spectrometer (Q-ToF Ultima, Micromass) with a 60 -min linear gradient of $0 \%-100 \%$ buffer B $(80 \%$ acetonitrile, $0.5 \%$ acetic acid) from a CAPLC HPLC system (Waters) at a flow rate of $\sim 170 \mathrm{~nL} / \mathrm{min}$. Combined peak lists were searched in the MSDB database using Mascot (http://www.matrixscience.com) allowing \pm 0.15 Da tolerance for both peptide and MS/MS fragment ion mass values.

\section{Immunodepletion and antibody addition}

Immunodepletion experiments were performed as described (Funabiki and Murray 2000). Briefly, anti-XErp1 or rabbit IgG antibodies were immobilized on Protein G beads (Dynal) in PBS buffer containing $0.1 \%$ Triton X-100 at $4^{\circ} \mathrm{C}$. Washed beads were mixed with the extract and incubated on ice with occasional mixing. Beads were retrieved on a magnet twice before the extract was warmed to $20^{\circ} \mathrm{C}$ for analysis of DNA and spindle morphology, and $\mathrm{H} 1$ kinase activity.

For antibody addition experiments, antibody was coupled to Protein G beads (Dynal) and incubated with a 10-fold molar excess of antigenic protein or buffer. Beads were washed extensively before mixing with extracts on ice. Extracts were then incubated at $20^{\circ} \mathrm{C}$.

\section{Immunoblotting}

Western blots were performed according to standard procedures. Briefly, for immunodetection of XErp1, samples were wet-blotted on PVDF membrane after PAGE. The membrane was blocked extensively and then incubated with $200 \mathrm{ng}$ affinitypurified XErp1 rabbit antibody. For Western blots against Xeno- pus, CDC27 and the Myc epitope-tag samples were blotted semi-dry on nitrocellulose membrane. The Myc epitope was detected with 9E10 hybridoma supernatant. As secondary antibodies, horseradish-peroxidase (HRP)-coupled anti-rabbit or anti-mouse antibodies were typically used at a concentration of $1 \mu \mathrm{g} / \mathrm{mL}$. Detection was carried out with Amersham ECL reagents or Pierce SuperSignal femto substrate.

\section{Kinase assays}

For analyses of Cdk1/cyclin B activity, H1 kinase assays were performed. The assay was started by mixing $1.62 \mu \mathrm{L}$ Xenopus extract with $18.6 \mu \mathrm{L}$ of kinase assay mix containing $8 \mu \mathrm{g}$ histone $\mathrm{H} 1,14 \mu \mathrm{M}$ ATP, and $6.5 \mu \mathrm{Ci} \gamma^{32} \mathrm{P}$-ATP in $\mathrm{H} 1$ buffer $(20 \mathrm{mM}$ $\beta$-glycerophosphate, $3 \mathrm{mM} \mathrm{MgCl}_{2}, 4 \mathrm{mM}$ EGTA, $0.025 \% \mathrm{NP} 40$ ). Reactions were carried out at room temperature and stopped by boiling samples in SDS-sample buffer. In vitro kinase assays using recombinant his-tagged Plx1 were performed as described (Descombes and Nigg 1998).

\section{In vitro ubiquitylation assays}

Yeast E1 enzyme was purchased from Boston Biochem Inc. E2 enzyme UbcX was expressed and purified from E. coli. Active APC/C was immunoprecipitated from " $\Delta 90$ extract" (Stemmann et al. 2001) using monoclonal anti-CDC27 antibodies (Sigma-Aldrich) immobilized on Protein G beads (Dynal). To obtain $\Delta 90$ extract, CSF extract was released from CSF arrest as described above and subsequently incubated with nondestructible cyclin B $\Delta 90$ to a final concentration of $5 \mu \mathrm{g} / \mathrm{mL}$. Assays were done as described (Kramer et al. 1998) except that $12 \mu \mathrm{g} / \mu \mathrm{L}$ UbcX was used and the buffer was CSF-XB. Briefly, his-tagged Cdc20 and MBP-tagged XErp1 proteins were preincubated with the immunoprecipitated APC/C in buffer QA (10 $\mathrm{mM}$ Tris- $\mathrm{HCl}$ at $\mathrm{pH} 7.5,100 \mathrm{mM} \mathrm{KCl}, 1 \mathrm{mM} \mathrm{MgCl}_{2}, 0.1 \mathrm{mM}$ $\mathrm{CaCl}_{2}, 1 \mathrm{mM}$ DTT) for $30 \mathrm{~min}$. The beads were washed once in $\mathrm{QA}$ and twice in CSF-XB, then the reaction was started by mixing the washed beads with a mix containing E1, E2, Ubiquitin, an energy regeneration system, and radiolabeled, in vitro translated $\mathrm{cycB}^{\mathrm{NT}}$. Samples were withdrawn after 0, 6, and $12 \mathrm{~min}$ and boiled in sample buffer.

\section{Acknowledgments}

We thank Francis Barr, Stefan Jentsch, Edgar Kramer, Ingmar Schön, and Olaf Stemmann for generously sharing reagents and for critical reading of the manuscript. We are grateful to Thierry Lorca, Yong Wan, and Stephan Geley for reagents, and Jenny Bormann and Samantha Wattam for excellent technical assistance. Research in the lab of T.U.M. is supported by Emmy Noether grant MA 1559/4-2 from the Deutschen Forschungsgemeinschaft.

\section{References}

Barr, F.A., Sillje, H.H., and Nigg, E.A. 2004. Polo-like kinases and the orchestration of cell division. Nat. Rev. Mol. Cell Biol. 5: 429-440.

Brassac, T., Castro, A., Lorca, T., Le Peuch, C., Doree, M., Labbe, J.C., and Galas, S. 2000. The polo-like kinase Plx1 prevents premature inactivation of the APC(Fizzy)-dependent pathway in the early Xenopus cell cycle. Oncogene 19: 3782-3790.

Desai, A., Murray, A., Mitchison, T.J., and Walczak, C.E. 1999. The use of Xenopus egg extracts to study mitotic spindle 
assembly and function in vitro. Methods Cell Biol. 61: 385412.

Descombes, P. and Nigg, E.A. 1998. The polo-like kinase Plx1 is required for $M$ phase exit and destruction of mitotic regulators in Xenopus egg extracts. EMBO J. 17: 1328-1335.

Donaldson, M.M., Tavares, A.A., Ohkura, H., Deak, P., and Glover, D.M. 2001. Metaphase arrest with centromere separation in polo mutants of Drosophila. J. Cell Biol. 153: 663676.

Dong, X., Zavitz, K.H., Thomas, B.J., Lin, M., Campbell, S., and Zipursky, S.L. 1997. Control of G1 in the developing Drosophila eye: rcal regulates Cyclin A. Genes \& Dev. 11: 94105.

Elia, A.E., Rellos, P., Haire, L.F., Chao, J.W., Ivins, F.J., Hoepker, K., Mohammad, D., Cantley, L.C., Smerdon, S.J., and Yaffe, M.B. 2003. The molecular basis for phosphodependent substrate targeting and regulation of Plks by the Polo-box domain. Cell 115: 83-95.

Fuchs, S.Y., Spiegelman, V.S., and Kumar, K.G. 2004. The many faces of $\beta$-TrCP E3 ubiquitin ligases: Reflections in the magic mirror of cancer. Oncogene 23: 2028-2036.

Funabiki, H. and Murray, A.W. 2000. The Xenopus chromokinesin Xkid is essential for metaphase chromosome alignment and must be degraded to allow anaphase chromosome movement. Cell 102: 411-424.

Gabrielli, B.G., Roy, L.M., and Maller, J.L. 1993. Requirement for Cdk2 in cytostatic factor-mediated metaphase II arrest. Science 259: 1766-1769.

Gietz, R.D. and Woods, R.A. 2002. Transformation of yeast by lithium acetate/single-stranded carrier DNA/polyethylene glycol method. Methods Enzymol. 350: 87-96.

Golan, A., Yudkovsky, Y., and Hershko, A. 2002. The cyclinubiquitin ligase activity of cyclosome/APC is jointly activated by protein kinases Cdk1-cyclin B and Plk. J. Biol. Chem. 277: 15552-15557.

Hansen, D.V., Loktev, A.V., Ban, K.H., and Jackson, P.K. 2004. Plk1 regulates activation of the anaphase promoting complex by phosphorylating and triggering SCF $\beta$ TrCP-dependent destruction of the APC inhibitor Emil. Mol. Biol. Cell 15: 5623-5634.

Kosako, H., Gotoh, Y., and Nishida, E. 1994. Mitogen-activated protein kinase kinase is required for the mos-induced metaphase arrest. J. Biol. Chem. 269: 28354-28358.

Kotani, S., Tugendreich, S., Fujii, M., Jorgensen, P.M., Watanabe, N., Hoog, C., Hieter, P., and Todokoro, K. 1998. PKA and MPF-activated polo-like kinase regulate anaphase-promoting complex activity and mitosis progression. Mol. Cell 1: 371-380.

Kraft, C., Herzog, F., Gieffers, C., Mechtler, K., Hagting, A., Pines, J., and Peters, J.M. 2003. Mitotic regulation of the human anaphase-promoting complex by phosphorylation. EMBO J. 22: 6598-6609.

Kramer, E.R., Gieffers, C., Holzl, G., Hengstschlager, M., and Peters, J.M. 1998. Activation of the human anaphase-promoting complex by proteins of the CDC20/Fizzy family. Curr. Biol. 8: 1207-1210.

Liu, J., Lewellyn, A.L., Chen, L.G., and Maller, J.L. 2004. The polo box is required for multiple functions of plx1 in mitosis. J. Biol. Chem. 279: 21367-21373.

Lorca, T., Cruzalegui, F.H., Fesquet, D., Cavadore, J.C., Mery, J., Means, A., and Doree, M. 1993. Calmodulin-dependent protein kinase II mediates inactivation of MPF and CSF upon fertilization of Xenopus eggs. Nature 366: 270-273.

Masui, Y. and Markert, C.L. 1971. Cytoplasmic control of nuclear behavior during meiotic maturation of frog oocytes. J. Exp. Zool. 177: 129-145.
Moshe, Y., Boulaire, J., Pagano, M., and Hershko, A. 2004. Role of Polo-like kinase in the degradation of early mitotic inhibitor 1, a regulator of the anaphase promoting complex/cyclosome. Proc. Natl. Acad. Sci. 101: 7937-7942.

Murray, A.W. 1991. Cell cycle extracts. Methods Cell Biol. 36. 2004. Recycling the cell cycle: Cyclins revisited. Cell 116: $221-234$.

Ohsumi, K., Koyanagi, A., Yamamoto, T.M., Gotoh, T., and Kishimoto, T. 2004. Emil-mediated M-phase arrest in Xenopus eggs is distinct from cytostatic factor arrest. Proc. Natl. Acad. Sci. 101: 12531-12536.

Rappsilber, J., Ishihama, Y., and Mann, M. 2003. Stop and go extraction tips for matrix-assisted laser desorption/ionization, nanoelectrospray, and LC/MS sample pretreatment in proteomics. Anal. Chem. 75: 663-670.

Regan-Reimann, J.D., Duong, Q.V., and Jackson, P.K. 1999. Identification of novel F-box proteins in Xenopus laevis. Curr. Biol. 9: R762-R763.

Reimann, J.D. and Jackson, P.K. 2002. Emil is required for cytostatic factor arrest in vertebrate eggs. Nature 416: 850 854.

Reimann, J.D., Freed, E., Hsu, J.Y., Kramer, E.R., Peters, J.M., and Jackson, P.K. 2001a. Emil is a mitotic regulator that interacts with Cdc20 and inhibits the anaphase promoting complex. Cell 105: 645-655.

Reimann, J.D., Gardner, B.E., Margottin-Goguet, F., and Jackson, P.K. 2001b. Emil regulates the anaphase-promoting complex by a different mechanism than Mad2 proteins. Genes \& Dev. 15: 3278-3285.

Sagata, N., Watanabe, N., Vande Woude, G.F., and Ikawa, Y. 1989. The c-mos proto-oncogene product is a cytostatic factor responsible for meiotic arrest in vertebrate eggs. Nature 342: 512-518.

Schwab, M.S., Roberts, B.T., Gross, S.D., Tunquist, B.J., Taieb, F.E., Lewellyn, A.L., and Maller, J.L. 2001. Bub1 is activated by the protein kinase p90(Rsk) during Xenopus oocyte maturation. Curr. Biol. 11: 141-150.

Shevchenko, A., Wilm, M., Vorm, O., and Mann, M. 1996. Mass spectrometric sequencing of proteins silver-stained polyacrylamide gels. Anal. Chem. 68: 850-858.

Shirayama, M., Zachariae, W., Ciosk, R., and Nasmyth, K. 1998. The Polo-like kinase Cdc5p and the WD-repeat protein Cdc20p/fizzy are regulators and substrates of the anaphase promoting complex in Saccharomyces cerevisiae. EMBO $T$. 17: 1336-1349.

Stemmann, O., Zou, H., Gerber, S.A., Gygi, S.P., and Kirschner, M.W. 2001. Dual inhibition of sister chromatid separation at metaphase. Cell 107: 715-726.

Sturgill, T.W., Ray, L.B., Erikson, E., and Maller, J.L. 1988. Insulin-stimulated MAP-2 kinase phosphorylates and activates ribosomal protein S6 kinase II. Nature 334: 715-718.

Tunquist, B.J. and Maller, J.L. 2003. Under arrest: Cytostatic factor (CSF)-mediated metaphase arrest in vertebrate eggs. Genes \& Dev. 17: 683-710.

Tunquist, B.J., Schwab, M.S., Chen, L.G., and Maller, J.L. 2002. The spindle checkpoint kinase bub1 and cyclin e/cdk2 both contribute to the establishment of meiotic metaphase arrest by cytostatic factor. Curr. Biol. 12: 1027-1033.

Tunquist, B.J., Eyers, P.A., Chen, L.G., Lewellyn, A.L., and Maller, J.L. 2003. Spindle checkpoint proteins Madl and $\mathrm{Mad} 2$ are required for cytostatic factor-mediated metaphase arrest. J. Cell Biol. 163: 1231-1242. 


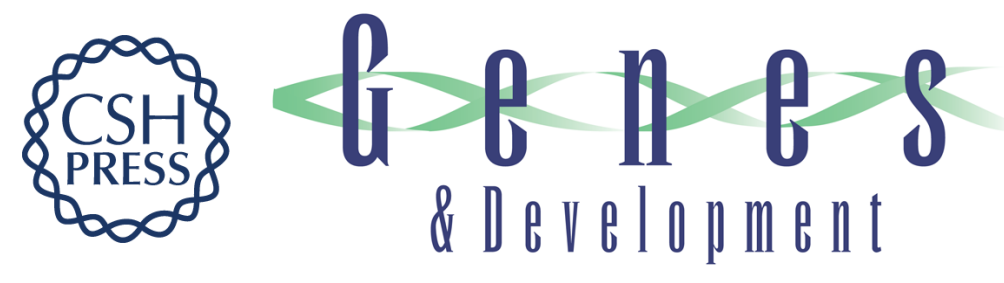

\section{Xenopus polo-like kinase PIx1 regulates XErp1, a novel inhibitor of APC/C activity}

Andreas Schmidt, Peter I. Duncan, Nadine R. Rauh, et al.

Genes Dev. 2005, 19:

Access the most recent version at doi:10.1101/gad.320705

$\begin{array}{ll}\text { References } & \text { This article cites } 37 \text { articles, } 15 \text { of which can be accessed free at: } \\ \text { http://genesdev.cshlp.org/content/19/4/502.full.html\#ref-list-1 }\end{array}$

License

Email Alerting

Receive free email alerts when new articles cite this article - sign up in the box at the top Service right corner of the article or click here.

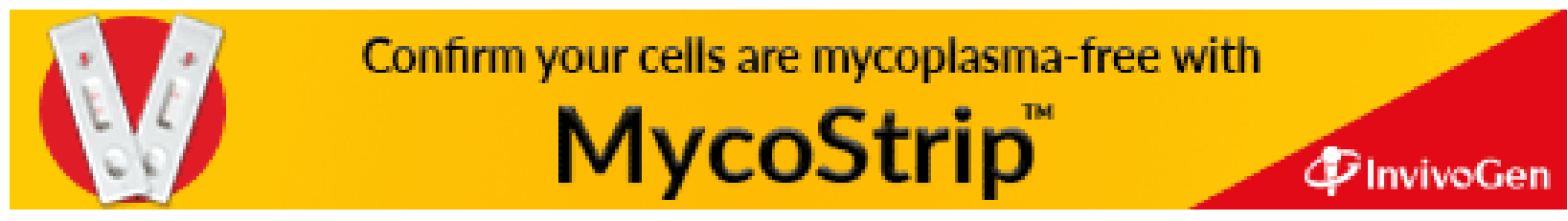

